\title{
Diaphragm strength in acute systemic lupus erythematosus in a patient with paradoxical abdominal motion and reduced lung volumes
}

\author{
P Hawkins, A G Davison, B Dasgupta, J Moxham
}

\begin{abstract}
Diaphragmatic weakness is reported as a common feature of the shrinking lung syndrome of systemic lupus erythematosus (SLE). However, in chronic stable SLE it has been shown that, despite poor performance of voluntary tests of diaphragm strength, twitch pressures obtained by stimulating the phrenic nerves are normal. We present a patient with acute SLE and pulmonary involvement who, despite having paradoxical abdominal motion and low maximal inspiratory pressures during voluntary manoeuvres, had normal diaphragm strength when assessed with magnetic stimulation of the phrenic nerves. Following immunosuppressive therapy symptoms and lung function improved, yet diaphragm contractility remained normal and unchanged. We suggest that this case supports the view that reduced diaphragm muscle contractility per se does not explain the small volume lungs and respiratory symptoms in patients with acute SLE.

(Thorax 2001;56:329-330)
\end{abstract}

Keywords: systemic lupus erythematosus; diaphragmatic strength; lung volumes

\section{Case history}

A 29 year old woman known to have systemic lupus erythematosus (SLE) was admitted for investigation of breathlessness and pyrexia. She had become increasingly breathless on exertion over the previous few weeks and had developed orthopnoea. She also complained of severe pleuritic chest pain which had been a feature of previous exacerbations of her SLE.

On examination the respiratory rate was 40 per minute, pulse 100 per minute, and

Accepted for publication

7 August 2000 paradoxical abdominal motion was present when sitting upright as well as when supine.

The chest radiograph showed small volume lungs associated with elevation of both hemidiaphragms but there were no pulmonary infiltrates. Arterial blood gas tensions on air showed a $\mathrm{PO}_{2}$ of $9.8 \mathrm{kPa}$ and a $\mathrm{PCO}_{2}$ of $4.4 \mathrm{kPa}$. The vital capacity (VC) was $1.431(40 \%$ predicted), total lung capacity (TLC) 2.081 ( $41 \%$ predicted), residual volume (RV) 0.651 (47\% predicted), and functional residual capacity (FRC) 1.131 (40\% predicted). The carbon monoxide transfer factor (TLCO) was reduced at $5.57 \mathrm{mmol} / \mathrm{kPa} / \mathrm{min}(20 \%$ predicted) and the transfer coefficient (KCO) was $4.93 \mathrm{mmol} / \mathrm{min} / \mathrm{kPa} / 1$ ( $88 \%$ predicted).

The patient was referred for respiratory muscle assessment because of suspected diaphragm weakness. Bilateral and unilateral anterior magnetic stimulation of the phrenic nerves was performed in addition to standard voluntary tests of respiratory muscle function, the results of which are shown in table 1 (visit 1 ).

She was treated with intravenous methylprednisolone (1 g) and cyclophosphamide (750 mg) every 15 days. There was a rapid improvement in her symptoms, particularly with regard to the pleuritic chest pain which, although still present, was markedly reduced during quiet breathing. Lung volumes remained reduced; VC 1.141 (32\% predicted), TLC 2.281 (45\% predicted), and FRC 1.541 (55\% predicted). ThCO was improved at $9.23 \mathrm{mmol} / \mathrm{kPa} / \mathrm{min}$ ( $34 \%$ predicted). The chest radiographic appearance was unchanged. Respiratory muscle assessment was repeated after 2 months of treatment (visit 2, table 1).

\section{Discussion}

Primary weakness of the diaphragm has been proposed as the cause of the loss of lung volume seen in some patients with SLE (the "shrinking lung" syndrome). ${ }^{1}$ However, early studies used volitional tests of inspiratory muscle strength ${ }^{2}$ which depend upon the subject being able to produce a maximal effort. Results from this laboratory using electrical stimulation of the phrenic nerves showed normal diaphragm strength in 12 patients with this syndrome. ${ }^{3}$ Magnetic stimulation is a recently introduced painless technique for stimulating the phrenic nerves and has proved 
useful for assessing diaphragm function in patients. ${ }^{4}$

Pressure measurements during the patient's acute illness demonstrated a reduction in gastric pressure (Pgas) during inspiration associated with paradoxical abdominal motion (fig 1A). This reflects failure of the diaphragm to partition the pressure generated during inspiration by the rib cage muscles, with consequent movement of the diaphragm into the thorax. This is followed by a normal diaphragm twitch response where Pgas is seen to increase as expected. A comparable pressure trace taken after 2 months of treatment (fig 1B) shows no reduction in Pgas during inspiration, and therefore no paradoxical abdominal motion, but Pgas does not rise normally which indicates that the diaphragm is not descending into the abdomen. Again, the twitch pressure response is normal. The reduction in Pdi during tidal breathing was present throughout the first assessment of this patient but was not apparent during the second assessment. As this inhibition of diaphragm contraction was absent during the twitch response, a voluntary reduction of diaphragm activation-possibly as a response to painwould explain this observation.

The small lung volumes observed are typical of the pattern seen in the shrinking lung syndrome but may be seen when interstitial lung disease is present. The reduction in FRC would require some fibrosis or basilar atelectasis, which would not necessarily be apparent on a plain chest radiograph. A high resolution CT scan of the lungs would be helpful in this respect. However, the relatively well preserved Kco combined with the clinical presentation make interstitial lung involvement unlikely in this case.

Orthopnoea is a common symptom in the acute SLE respiratory syndrome. Paradoxical abdominal motion has been described before in a patient with this condition but was attributed to diaphragm weakness by the authors who measured transdiaphragmatic pressure during a maximal voluntary inspiratory manoeuvre. $^{5}$

Pleuritic pain is a major feature of SLE and was prominent in our patient. It is probable that this pain prevented the patient from
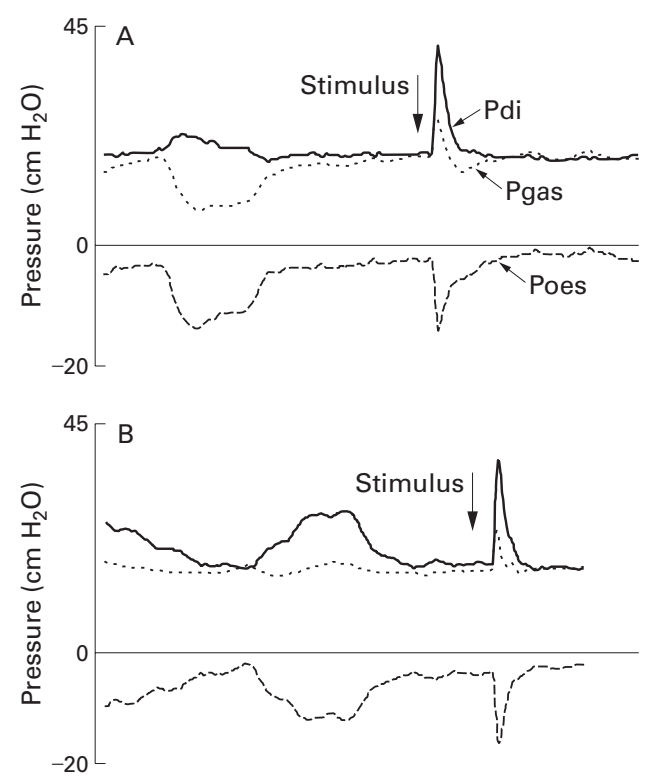

Figure 1 Intrathoracic and intra-abdominal pressures during spontaneous breathing recorded with the patient seated $(A)$ during the acute illness and (B) after treatment.

performing the voluntary tests of inspiratory muscle strength maximally. When the pain had improved after treatment she was able to produce somewhat greater pressures. Diaphragm strength, as assessed by phrenic nerve stimulation, was normal and did not change. We conclude that reduced diaphragm muscle contractility does not explain the small lung volumes in acute SLE. Inhibition of diaphragm activation, with pain being an important factor in some cases, could explain the observed diaphragm dysfunction.

1 Murin S, Wiedemann HP, Matthay RA. Pulmonary manifestations of systemic lupus erythematosus. Clin Chest Med 1998;19:641-65.

2 Gibson G, Edmonds J, Hughes G. Diaphragm function and lung involvement in systemic lupus erythematosus. Am $\mathcal{f}$ Med 1977;63:926-32.

3 Laroche C, Mulvey D, Hawkins P, et al. Diaphragm strength in the shrinking lung syndrome of systemic lupus erythematosus. Qf Med 1989;71:429-39.

4 Mills GH, Kyroussis D, Hamnegard C-H, et al. Bilateral magnetic stimulation of the phrenic nerves from an anterolateral approach. Am f Respir Crit Care Med 1996;154: 1099-105.

5 Thompson P, Dhillon D, Ledingham J, et al. Shrinking thematosus. Am Rev Respir Dis 1985;132:926-8. 